\section{Millimeter-Wave High-Gain SIW End-Fire Bow-tie Antenna}

\author{
Abdolmehdi Dadgarpour, Behnam Zarghooni, Bal S.Virdee, and \\ Tayeb A.Denidni
}

\begin{abstract}
This paper presents a high gain bow-tie antenna that operates across $57 \mathrm{GHz}-64 \mathrm{GHz}$ for application in high data rate point-to-point communication systems. The proposed antenna consists of a pair of bow-tie radiators, where each radiator is etched on the opposite side of the common dielectric substrate and fed through substrate integrated waveguide (SIW) feed-line. The bow-tie radiators are arranged to cross each other symmetrically by tilting the feed-lines by 30 degrees in order to enhance the antenna gain and to obtain the required radiation pattern. The antenna is loaded with a pair of double G-shaped resonators (DGR) that are located in a region between the radiators and SIW in order to suppress the back-lobe level in the H-plane. Embedded in the E-plane of the antenna is an array of zero-index metamaterial (ZIM) unit-cells whose purpose is to effectively confine the electromagnetic waves in the end-fire direction in order to enhance its gain performance. A prototype antenna was fabricated and its performance was measured to validate the simulation results. The proposed structure exhibits a gain of 11.8 $12.5 \mathrm{dBi}$ over the frequency range of $57 \mathrm{GHz}-64 \mathrm{GHz}$ with reflection-coefficient less than $-11 \mathrm{~dB}$. In addition, the proposed antenna exhibits good cross-polarization which is less than $-17 \mathrm{~dB}$ in both E- and H-planes at $60 \mathrm{GHz}$.
\end{abstract}

IndexTerms-Millimeter-wave antenna, zero-index metamaterial, bow-tie antenna, end-fire antenna.

\section{INTRODUCTION}

The unlicensed industrial, scientific and medical (ISM) band centered at $60 \mathrm{GHz}(57 \mathrm{GHz}-64 \mathrm{GHz})$ provides a large bandwidth of $7 \mathrm{GHz}$ however signals at these frequencies incur high loss due to atmospheric absorption. These characteristics make it suitable for high data rate (in the order of multiples of Gbps) applications over a short-range including the transmission of uncompressed high definition video streaming and wireless point-to-multipoint connections. High propagation loss at these frequencies makes the design of high-gain antennas crucial. For such antennas to be commercially viable they need to be compatible with low cost technologies. Numerous antenna designs at $60 \mathrm{GHz}$ have been previously proposed and implemented using multi-layer technology including low temperature co-fired ceramic (LTCC), liquid crystal polymer (LCP) and high end hydrocarbon ceramic printed circuit boards (PCB) [1]-[6]. In [7] the authors have described a high gain antenna operating at $60 \mathrm{GHz}$ using dielectric resonator antenna (DRA) which is surrounded with an electromagnetic band-gap (EBG) structure to provide a maximum peak gain of $8.7 \mathrm{dBi}$. The aforementioned antenna designs are complex and expensive to fabricate. In addition, they have a broadside radiation pattern that restricts their application in beam-switching systems.

A. Dadgarpour, B. Zarghooni and T. A. Denidni are with INRS-EMT University of Quebec, Montreal, Quebec, CANADA. (Email: abdolmehdi.dadgarpour@emt.inrs.ca, zarghooni@emt.inrs.ca, denidni@emt.inrs.ca). B. S. Virdee is with Center for Communications Technology at London Metropolitan University, UK.
In this paper a planar bow-tie antenna is presented that uses an amalgamation of different techniques to realize a high gain structure for operation over $57 \mathrm{GHz}-64 \mathrm{GHz}$. Work in this paper is inspired by reference [8]. Configuration of the proposed antenna, shown in Fig.1, comprises a pair of bow-tie radiators. Each radiator is etched on the opposite sides of the substrate and arranged to cross each other symmetrically. The radiators are tilted by 30 degrees with respect to the end-fire direction $(+y)$ to increase the antenna gain and determine its radiation pattern. The antenna is loaded with double G-shaped resonators (DGR) that have a band-stop property. The two DGR structures are located in the vicinity of the bow-tie radiators and SIW in order to suppress the back-lobe level in the H-plane. An array of zero-index metamaterial (ZIM) unitcells is embedded laterally in the E-plane above the bow-tie radiators to convert the spherical waves into uniform field distribution. The fabricated bow-tie antenna provides a gain of 11.5-12 dBi over $57 \mathrm{GHz}-64 \mathrm{GHz}$, and a reflectioncoefficient of less than $-11 \mathrm{~dB}$. Although the proposed antenna exhibits a comparable gain performance to previously reported millimeter-wave antenna designs [1]-[7], it has the advantage of being a simpler structure that is cost effective to fabricate.

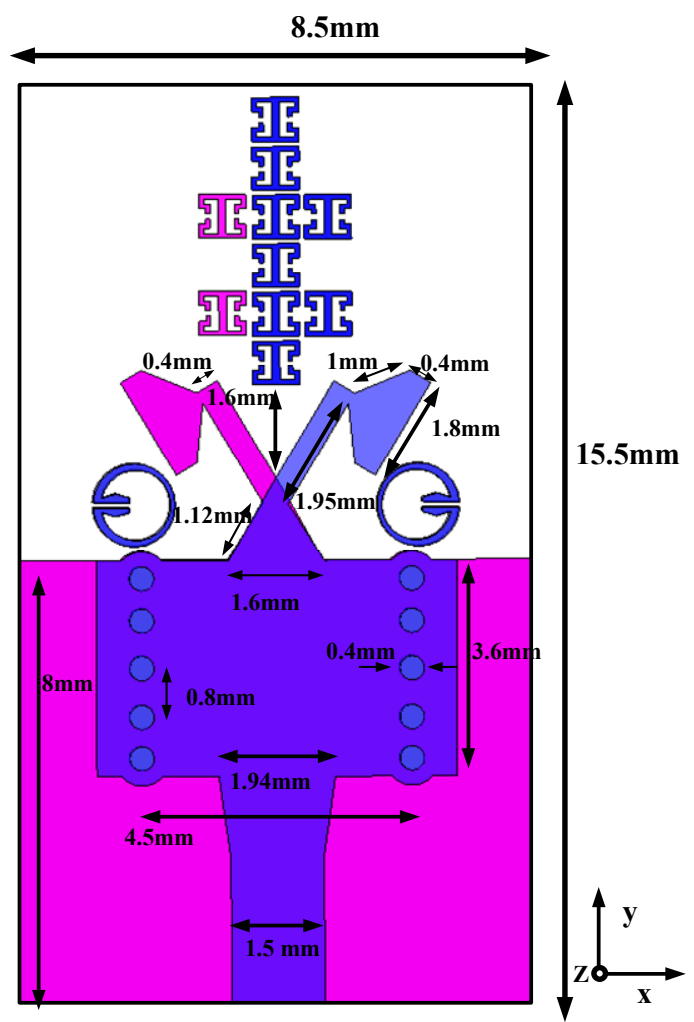

Fig. 1. Configuration of the proposed tilted bow-tie antenna with DGR and an array of ZIM unit-cells.

In the following section, we first propose the initial design of the bow-tie antenna with SIW feed-line. The antenna's characteristics are then investigated when the bow-tie radiator is tilted with respect to the end-fire direction. It is shown that when the antenna is loaded with double G-shaped resonators its back-lobe level in the H-plane and gain are enhanced. Lastly, we propose a zero-index metamaterial unit-cell structure. An arrangement of these ZIM unit-cells are 
embedded laterally in the E-plane of the tilted bow-tie antenna to realize a high gain response over $57 \mathrm{GHz}-64 \mathrm{GHz}$.

\section{ANTENNA DESIGN}

The geometry of the proposed antenna in Fig. 1 consists of a SIW structure resembling a rectangular tunnel created between the top and bottom copper foils of the dielectric substrate that is surrounded by the two rows of metallic vias. The tunnel is equivalent to a dielectric-filled rectangular waveguide, where the two rows of metallic vias are equivalent to its side walls. The SIW configuration that is depicted in Fig. 1 is a modified version described in [9]. This configuration creates a 180 degree phase difference between the currents along the top and bottom layers of the SIW structure. The lower end of the SIW structure is tapered to a microstrip feed-line, and the upper end tapered to a bow-tie radiating element. The bottom of the substrate comprises a truncated ground plane with an identical bow-tie antenna that is arranged to symmetrically cross with the dipole antenna on the top metallic layer of the substrate.

The SIW structure was fabricated on RT 5880 dielectric substrate with relative permittivity of 2.2 , thickness of $0.5 \mathrm{~mm}$ and loss tangent of 0.0009 . The feed-line to the SIW structure has a tapered transition to provide a good impedance match over the required frequency range of $57 \mathrm{GHz}-64 \mathrm{GHz}$. The normalized E-plane $(x y)$ and $\mathrm{H}$-plane $(y z)$ radiation patterns of this structure at $60 \mathrm{GHz}$ are shown in Fig. 2. The worst case back-lobe level (BLL) in H-plane is about $-0.6 \mathrm{~dB}$ which is not appropriate for practical purposes. The worst case sidelobe level (SLL) in the E-plane $(x y)$ is about $-7 \mathrm{~dB}$. Improving the BLL in the H-plane will result in more power being directed into the end-fire direction. Since the antenna gain is inversely related to its beamwidth, so improving the SLL in the E-plane $(x y)$ will result in a narrower beamwidth and enhanced gain performance.

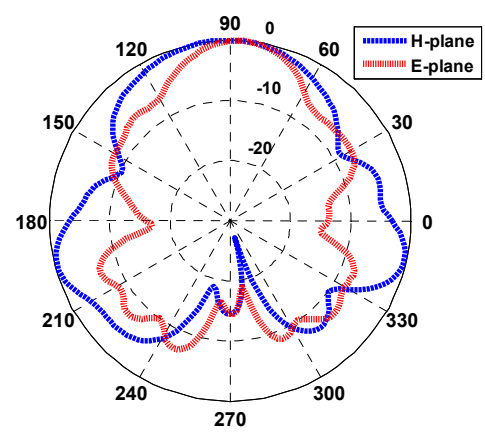

Fig. 2. The normalized radiation patterns of the un-tilted bow-tie antenna in the E- and H-planes at $60 \mathrm{GHz}$. Angle is in degrees and radius in $\mathrm{dB}$.

\section{The Effect of Bow-tie Radiator Tilt Angle}

The tilt angle of the bow-tie radiators with respect to $y$-axis was investigated. The results of this study in Fig. 3 show that increasing the tilt angle from 5 to 30 degrees has a direct effect on the antenna gain. Certain tilt angles improve the antenna gain over the frequency range of $56 \mathrm{GHz}-64 \mathrm{GHz}$, which is equivalent to enlargement of the antenna aperture. A tilt angle of 30 degrees is observed to provide an optimum gain performance. At a tilt angle of 30 degrees, the gain varies between 7-8.8 dB, which corresponds to a gain enhancement of $1.5-2.0 \mathrm{~dB}$ over frequency band of $57 \mathrm{GHz}-64 \mathrm{GHz}$ compared with the antenna with 0 degree tilt angle.

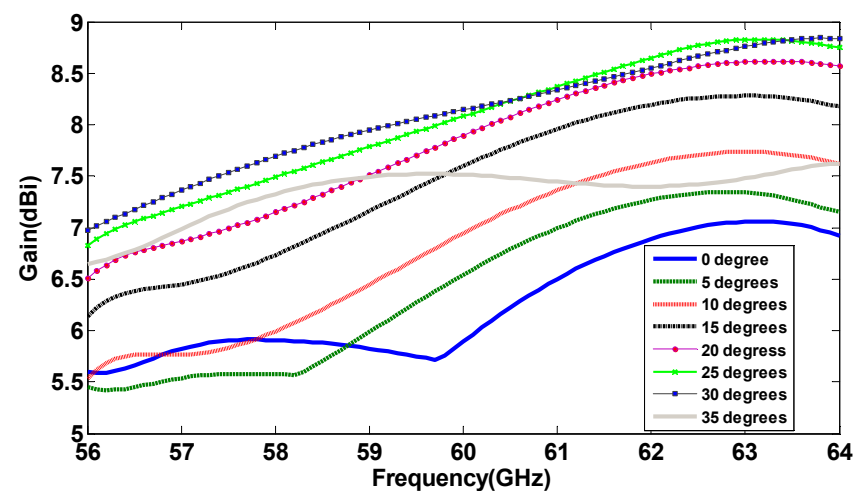

Fig. 3. Antenna gain response as a function of bow-tie tilt angle.

The radiation patterns of the antenna with the bow-tie radiators tilted by 30 degrees are shown in Fig. 4. This result shows there is a distinct improvement in the SLL in both Eplane $(x y)$ and H-plane $(y z)$ however there is degradation in BLL compared with the un-tilted radiators in Fig. 2.

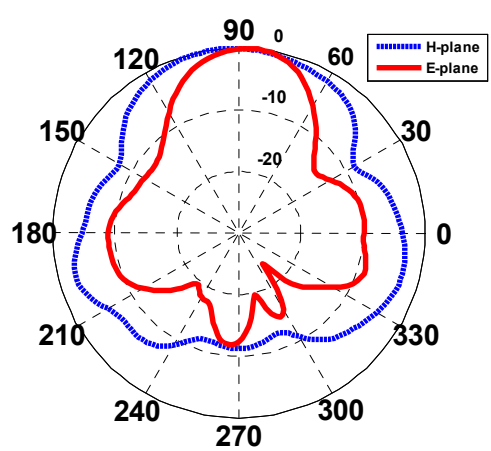

Fig. 4. The normalized radiation patterns of the tilted bow-tie antenna in the $\mathrm{E}-$ and $\mathrm{H}-$ planes at $60 \mathrm{GHz}$. Angle is in degrees and radius in $\mathrm{dB}$.

\section{EFFECT OF DGR LOADING}

In order to reduce the back-lobe radiation in the H-plane $(y z)$, the double G-shaped resonator, shown in Fig. 5, was employed. This resonator is based on the broadside coupled split-ring resonator structure described in [10]. The DGR structure is constructed on both layers of the substrate in the vicinity of the bow-tie radiating elements. This is necessary to couple the DGR with the electromagnetic field emanating from the radiating elements in order to induce current along the perimeter of DGR structure. The DGR behaves equivalently to an LC resonant circuit which is excited by an EM wave source.

The S-parameters of the DGR structure in Fig. 5 were established by applying the ports on both sides of the resonator along the $x y$-plane. Application of a plane wave along the $y$-axis results in the electric and magnetic field polarized in the $x$ - and $z$-axis, respectively. 
The S-parameter response of the DGR structure in Fig. 6 exhibits a wide band stop response over $55.4 \mathrm{GHz}-65 \mathrm{GHz}$ for a rejection level greater than $-10 \mathrm{~dB}$. Embedding the proposed DGR structure on both sides of the substrate and next to the tilted bow-tie radiators, results in reduction of the BLL. In the $\mathrm{H}$-plane $(y z) \mathrm{BLL}$ is reduced by about $4 \mathrm{~dB}$ and in the E-plane $(x y)$ by about $3 \mathrm{~dB}$, as shown in Fig. 7. The E-field distribution over the antenna, which is shown in Fig. 8, clearly demonstrates there is a reduction in the E-field intensity at the sides of the tilted bow-tie radiators in the locality of the DGR structures.

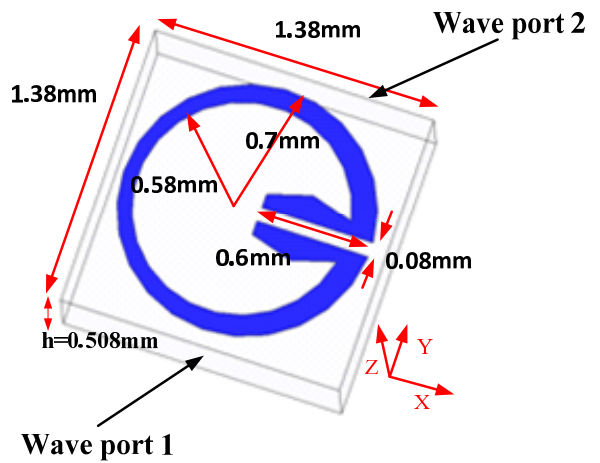

Fig. 5. The structure of the double G-shaped resonator.

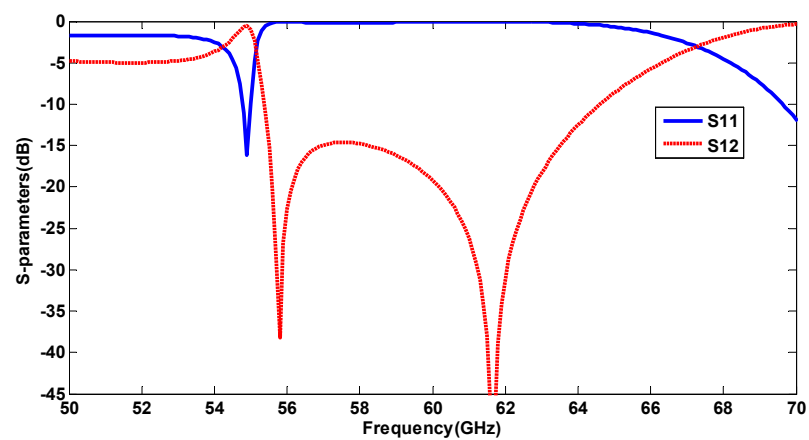

Fig. 6. S-parameter $\left(\left|S_{12}\right|\right.$ and $\left.\left|S_{11}\right|\right)$ response of the DGR configuration

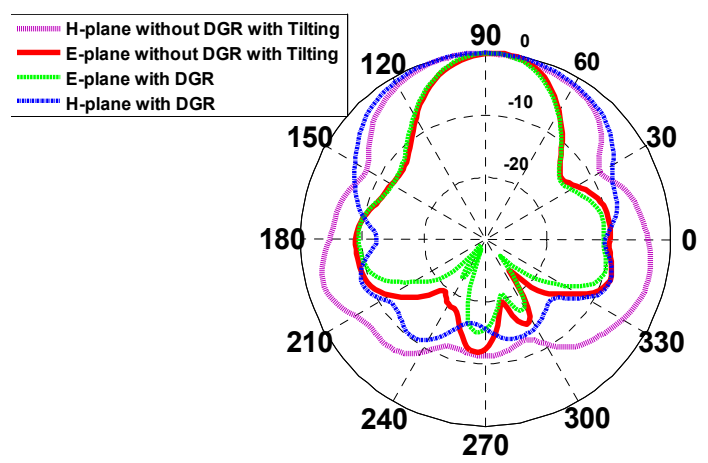

Fig. 7. The normalized radiation patterns of the proposed antenna with and without DGR loading in the E-and H-planes. Angle is in degrees and radius in dB.

With DGR loading, the antenna gain varies from 9.57 to $8.14 \mathrm{~dB}$ over $57 \mathrm{GHz}-64 \mathrm{GHz}$, as shown in Fig. 9. Gain enhancement of 2.14-0.28 dB is observed across $57 \mathrm{GHz}-62$ $\mathrm{GHz}$ compared with the bow-tie antenna without DGR loading. However, above $62.5 \mathrm{GHz}$ the gain begins to decline and reduces by $0.7 \mathrm{~dB}$ at $64 \mathrm{GHz}$.

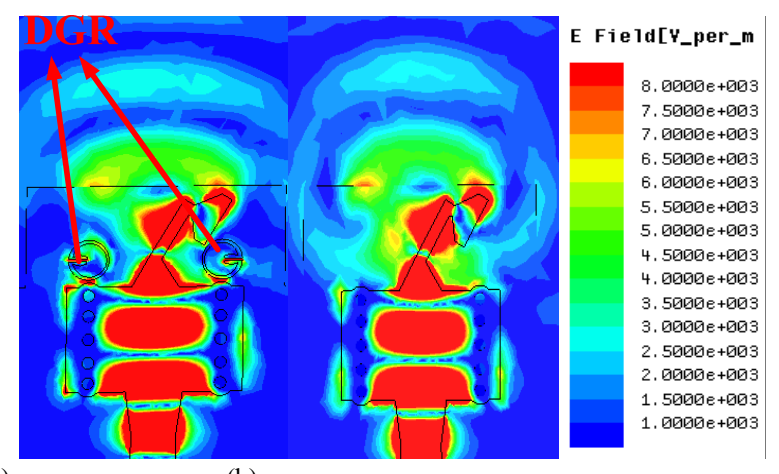

(a)

(b)

Fig. 8. The E-field distribution over the tilted antenna: (a) with DGR loading, and (b) without DGR loading.

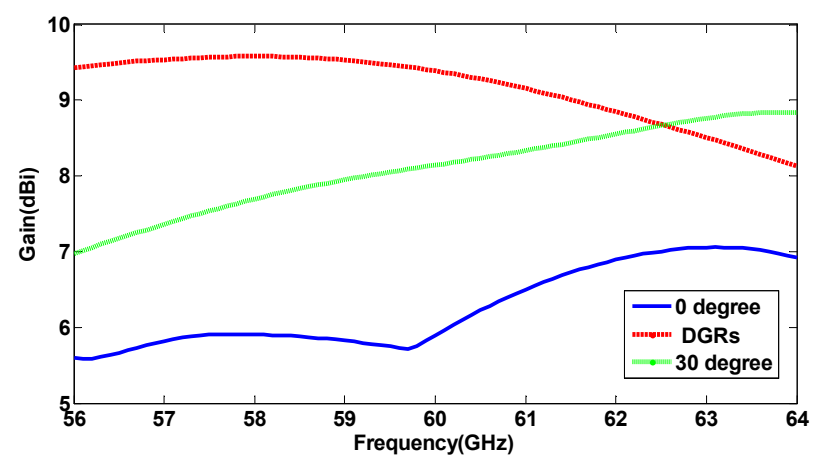

Fig. 9. Comparison of bow-tie antenna gain for un-tilted and tilted radiators, and the antenna gain for a tilted bow-tie antenna with DGR loading.

\section{ZIM UNIT-CELL}

In order to enhance the gain of the antenna a zero-index metamaterial unit-cell structure, shown in Fig. 10, was embedded in the antenna. The ZIM unit-cell structure is a modified version of the electric LC resonator described in [11]. Here we have increased the effective inductance and capacitance of the structure so that its resonant frequency is lower and it exhibits near zero permittivity over frequency range $57 \mathrm{GHz}-64 \mathrm{GHz}$.

The intrinsic electrical parameters of the ZIM unit-cell (effective permittivity and permeability) were determined using a standard extraction algorithm which is described in [12] and is based on the S-parameters. This involves assigning a perfect electric conducting (PEC) and the perfect magnetic conducting (PMC) boundary conditions in the $x z$-plane and $x y$ plane to the ZIM structure in Fig. 10. The transmission and reflection-coefficient response of the ZIM unit-cell in Fig. 11 shows the signal is uninhibited to pass through the structure over the required operating frequency range of $57 \mathrm{GHz}-64$ $\mathrm{GHz}$.

The extracted effective permittivity, permeability and refractive-index of the proposed ZIM unit-cell are shown in Fig. 12. The simulation results show that the refractive-index of the ZIM unit-cell is less than unity over the frequency range 
of $55 \mathrm{GHz}-65 \mathrm{GHz}$. It also shows the permittivity is less than 0.75 over the frequency range of $55 \mathrm{GHz}-64 \mathrm{GHz}$.

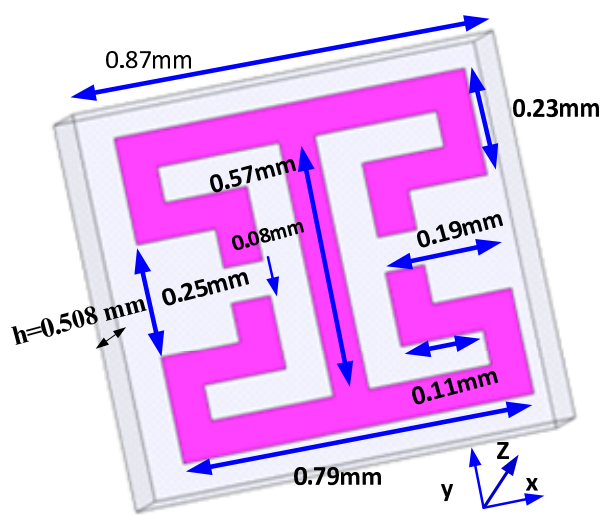

Fig.10. The configuration of the proposed ZIM unit-cell.

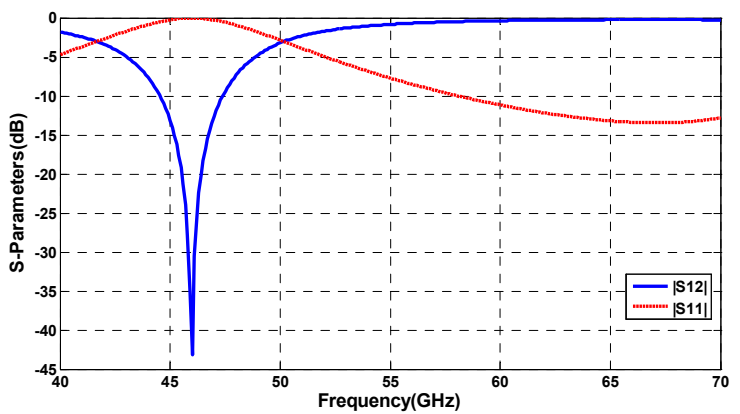

Fig. 11.S-parameter $\left(\left|S_{12}\right|\right.$ and $\left.\left|S_{11}\right|\right)$ response of the proposed ZIM bow-tie antenna.

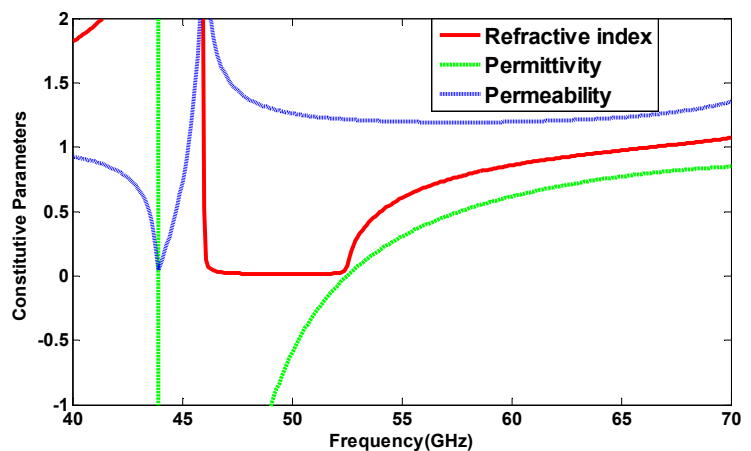

Fig. 12. Retrieved characterizing parameters of the ZIM unit-cell, i.e. permittivity, permeability and refractive-index.

\section{ANTENNA With ARRAY OF ZIM UNIT-CELlS}

In this section, various combinations of ZIM unit-cell arrangements etched on the antenna are investigated. The ZIM unit-cells are embedded laterally in the E-plane above the tilted bow-tie antenna which is loaded with DGR structures. The initial antenna design is loaded with the number of ZIM unit-cells $(1,3,5)$ from the bow-tie antenna to $+y$ direction. The antenna gain of this array configuration varies between 11.8$11.3 \mathrm{~dB}$ over $57 \mathrm{GHz}-64 \mathrm{GHz}$, as shown in Fig. 13. An array configuration of $(1,3,1,3,1,1)$ ZIM unit-cells provides again variation of 11.8-12.5 dB between 57-64 GHz, which corresponds to an average gain enhancement of $3.95 \mathrm{~dB}$ compared with the tilted bow-tie antenna without ZIM unitcells. An array configuration of $(1,3,1,3,1,1)$ ZIM unit-cells is used in the final antenna design.

The radiation patterns in the E-plane $(x y)$ and H-plane $(y z)$ of the final antenna consisting of tilted bow-tie radiators with embedded DGR structures and ZIM unit-cell arrays are shown in Fig. 14. Compared with the radiation patterns of the initial bow-tie antenna in Fig. 2, it can be observed that the side-lobe and back-lobe level in both planes are improved considerably by approximately $10 \mathrm{~dB}$.

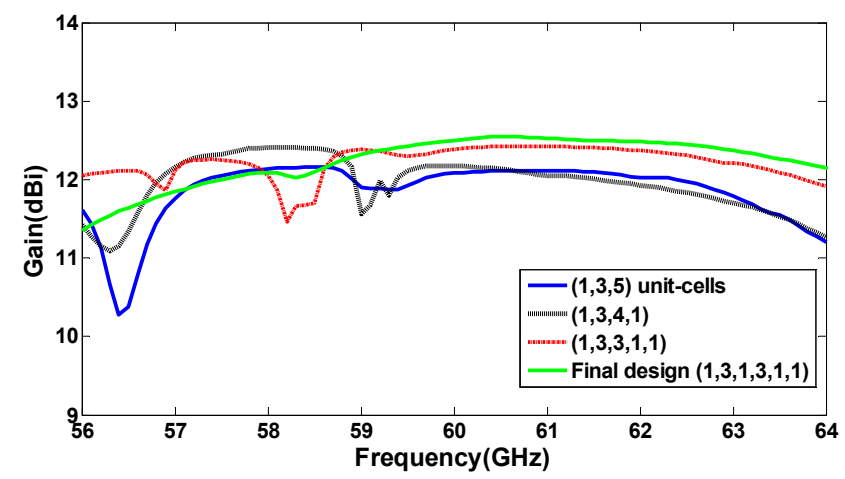

Fig. 13. Antenna gain with various ZIM unit-cell arrangements.

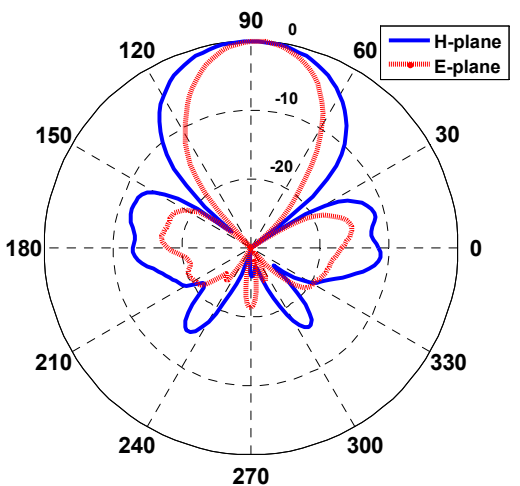

Fig. 14. The normalized radiation patterns of the final antenna in the E- and $\mathrm{H}$-planes at $60 \mathrm{GHz}$. Angle is in degrees and radius in $\mathrm{dB}$.

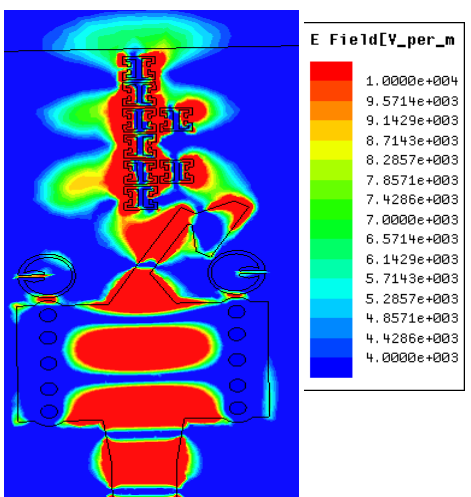

Fig. 15. The E-field distribution over the antenna at $60 \mathrm{GHz}$. 
The E-field distribution over the final antenna in Fig.15 confirms that the DGR and ZIM unit-cell loading has a great impact on confining the radiation beam to end-fire direction and suppressing the antenna'sside and back-lobes.

\section{EXPERIMENTAL RESULTS}

The proposed tilted bow-tie antenna incorporating an array of ZIM unit-cells and a pair of DGR structures was fabricated and its performance was measured. The photograph of the fabricated prototype antenna is shown in Fig. 16. The magnitude of S11 was measured using an Anritsu 3739C Vector Network Analyzer. The $1.85 \mathrm{~mm}$ end-launch connector was utilized to measure the reflection-coefficient. The measured reflection-coefficient of the antenna, shown in Fig. 17 , is less than $-15 \mathrm{~dB}$ over $59 \mathrm{GHz}-64 \mathrm{GHz}$.

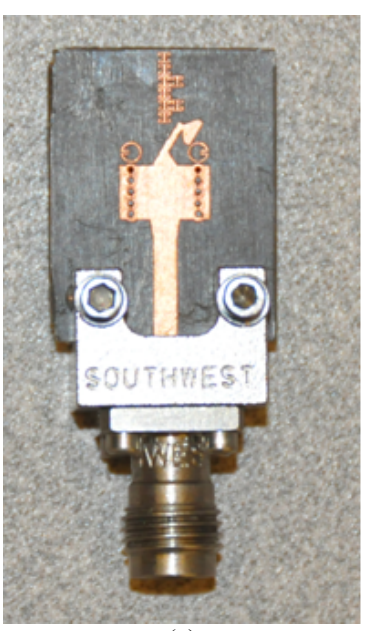

(a)

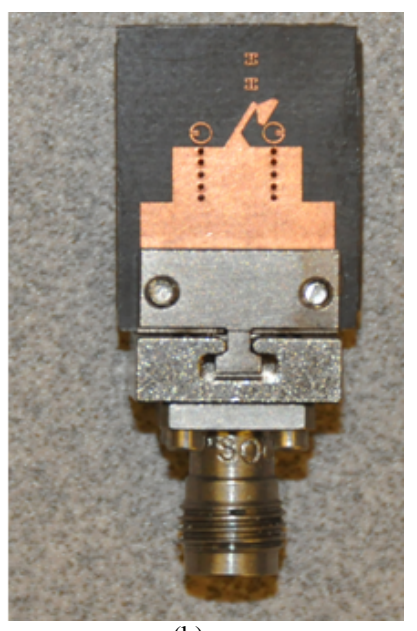

(b)
Fig. 16. Photograph of the fabricated prototype antenna, (a) top, and (b) bottom.

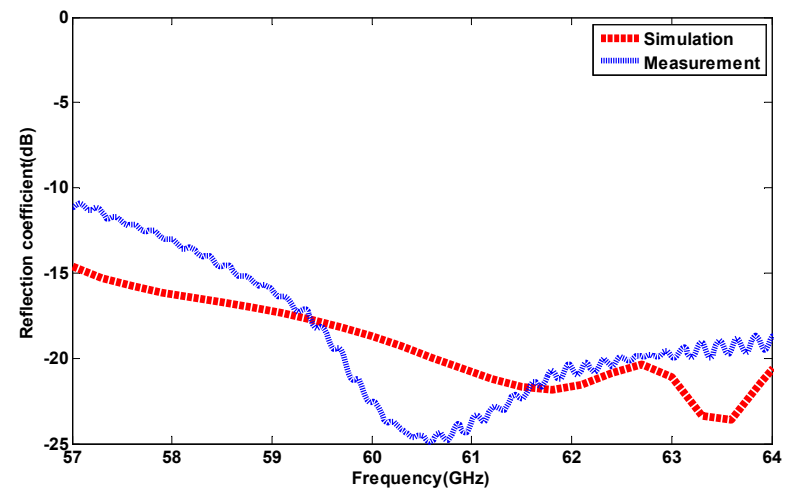

Fig. 17.Measured and simulated reflection-coefficient of the ZIM tilted bowtie antenna.

The radiation patterns were measured using MI Technology anechoic chamber using the antenna test arrangement shown in Fig. 18, where a horn antenna was located at the focal point of the reflector to generate a plane wave towards the antenna under test. The measured radiation-patterns of the ZIM bowtie antenna in the E-plane $(x y)$ and H-plane $(y z)$ at 58, 60, 62 and $64 \mathrm{GHz}$ are plotted in Fig. 19 and 20. Correlation between the simulated and measured results is good. The results indicate that the side-lobe level of the antenna in the H-plane is less than $-11.5 \mathrm{~dB}$ at $60 \mathrm{GHz}$ and less than $-9.7 \mathrm{~dB}$ at 64 $\mathrm{GHz}$, which is better than that of Yagi-Uda design in [13] that has SLL of $-5 \mathrm{~dB}$; and the front to back radiation in both planes is better than $-20 \mathrm{~dB}$. Additionally, the crosspolarization of the antenna in the E-plane $(x y)$ and H-plane $(y z)$ is better than $-17 \mathrm{~dB}$ at $60 \mathrm{GHz}$, which is better than ZIM Vivaldi antenna in [8] with $-10 \mathrm{~dB}$ cross polarization. The measured radiation efficiency of the antenna is $91 \%$ at 60 GHz.

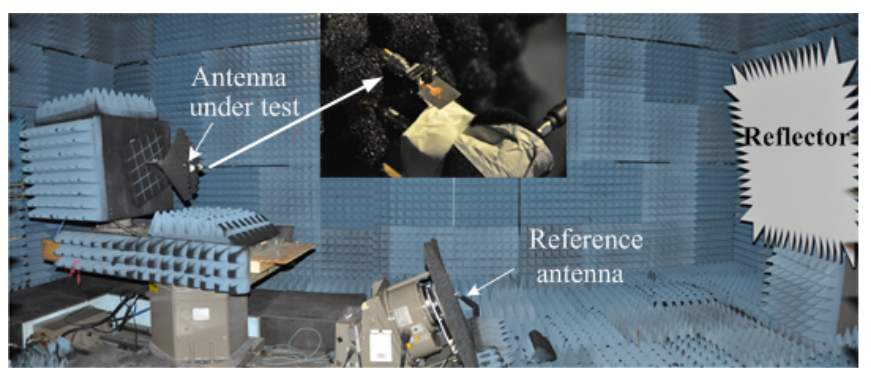

Fig. 18. Photograph of the antenna measurement set-up.

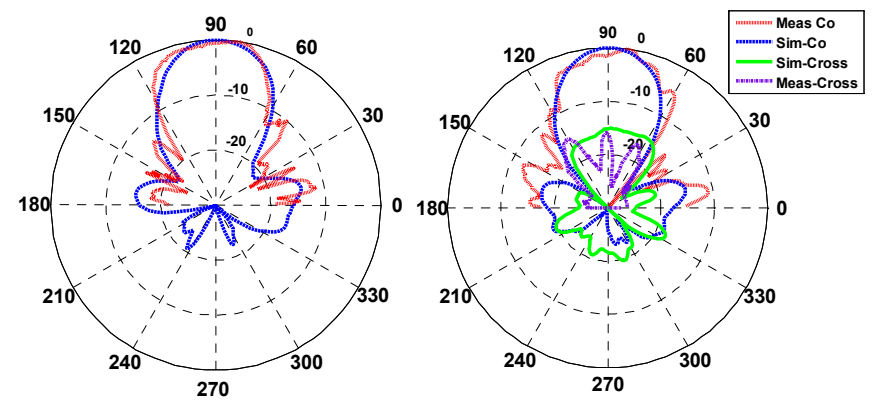

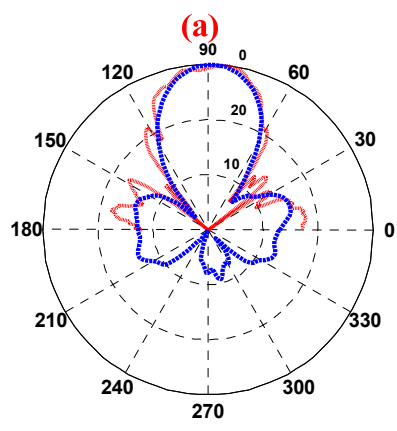

(c)

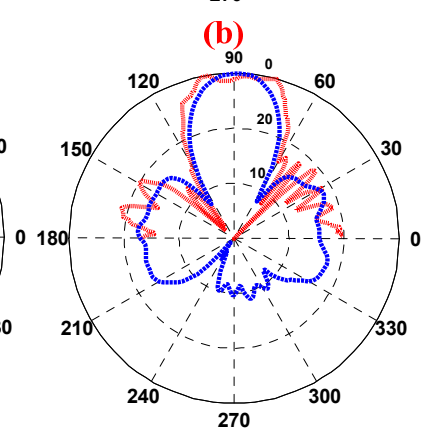

(d)
Fig. 19. Normalized simulated and measured radiation patterns of the proposed antenna in the E-plane $(x-y)$ at: (a) $58 \mathrm{GHz}$, (b) $60 \mathrm{GHz}$ (c), $62 \mathrm{GHz}$, and (d) $64 \mathrm{GHz}$. Angle is in degrees and radius in $\mathrm{dB}$.

The antenna gain was measured using the comparative method that involves measuring the signals received by the reference horn antenna and the test antenna, and determining the relative difference in the gain of both antennas. The measured loss due to SIW and $1.85 \mathrm{~mm}$ end-launch connectors, shown in Fig. 21, is $2-2.5 \mathrm{~dB}$ over the frequency range of $57 \mathrm{GHz}-64 \mathrm{GHz}$. This loss was taken into account in the gain measurement. It is important to mention that in the simulation we have used wave ports on both ends of the SIW structure shown in Fig. 21, and the simulated antenna gain takes into account the SIW loss. 


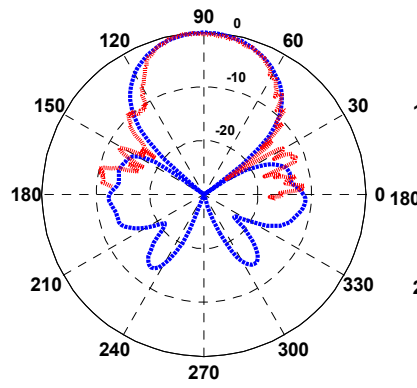

(a)

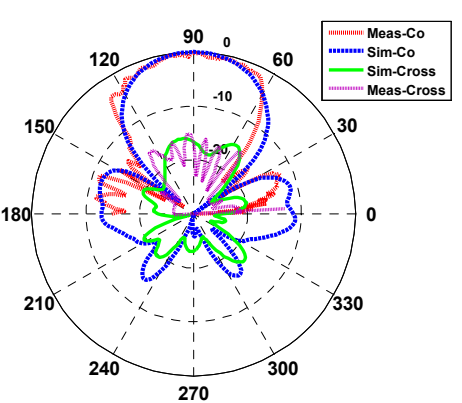

(b)

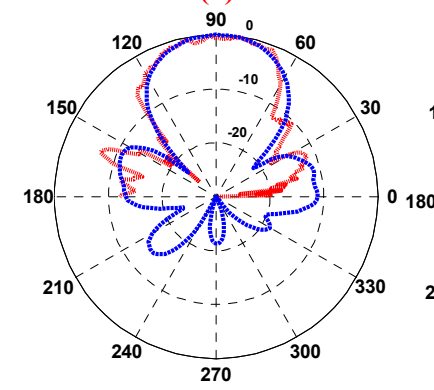

(c)

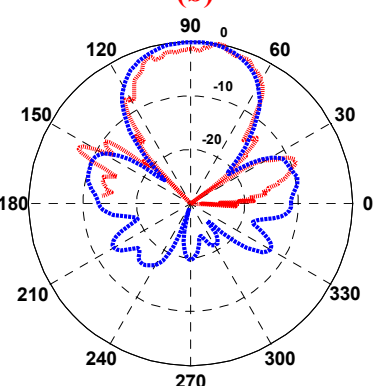

(d)
Fig. 20. Normalized simulated and measured radiation patterns of the proposed antenna in the H-plane (yz) at: (a) $58 \mathrm{GHz}$, (b) $60 \mathrm{GHz}$, (c) $62 \mathrm{GHz}$ (d), and $64 \mathrm{GHz}$. Angle is in degrees and radius in $\mathrm{dB}$.

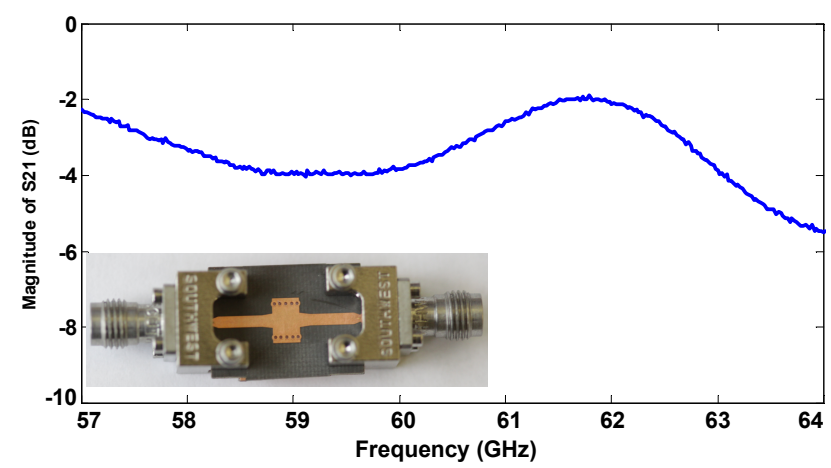

Fig. 21.Measured insertion-loss of back-to-back end-launch connectors along with SIW microstrip line.

The measured peak gains of the proposed antenna are given in Table I. The discrepancy between simulation and measurement results is about $0.5 \mathrm{~dB}$.

Table I. Measured and simulated gain of the proposed tilted bow-tie antenna at various frequencies

\begin{tabular}{|c|c|c|}
\hline Freq. (GHz) & Simulated peak gain $(\mathrm{dB})$ & Measured peak gain $(\mathrm{dB})$ \\
\hline 58 & 11.9 & 11.5 \\
\hline 60 & 12.4 & 12.0 \\
\hline 62 & 12.5 & 12.0 \\
\hline 64 & 12.1 & 11.7 \\
\hline
\end{tabular}

\section{CONCLUSION}

A modified bow-tie antenna structure is proposed for high gain performance across $57 \mathrm{GHz}-64 \mathrm{GHz}$. The antenna consists of a pair of tilted bow-tie radiators, where each radiator is etched on the opposite side of the common dielectric substrate and fed through substrate integrated waveguide (SIW) feed-line. The bow-tie radiators are arranged to cross each other symmetrically in order to enhance the antenna gain and to obtain the required radiation pattern. It is shown that the antenna gain can be significantly enhanced by loading the antenna with an array of ZIM unit-cells that are implemented laterally to the radiators. In addition, double Gshaped resonators are employed to reduce the back-lobe level of the antenna. The antenna exhibits a measured gain of 11.5$12 \mathrm{dBi}$ over frequency range of $57 \mathrm{GHz}-64 \mathrm{GHz}$ with reflection-coefficient less than $-11 \mathrm{~dB}$. The proposed antenna is simple to design and inexpensive to fabricate. The characteristics of the antenna make it suitable for application in $60 \mathrm{GHz}$ indoor wireless communication systems.

\section{REFERENCES}

[1] H-L. Wang, Y-X. Guo, W-X. Sheng, "Wideband high-gain 60-GHz LTCC L-probe patch antenna array with a soft surface," IEEE Trans. Antennas Propagat., vol.61, no.1, pp.153-161, Jan.2013.

[2] H.Chu, Y-X Guo, Z.Wang, "60-GHz LTCC wideband vertical off-center dipole antenna and arrays," IEEE Trans. Antennas Propagat., vol.61, no.1, pp.153-161, Jan.2013.

[3] K. Gong, Z.N. Chen, X. Qing, P. Chen, W. Hong, "Substrate integrated waveguide cavity-backed wide slot antenna for $60-\mathrm{GHz}$ bands," IEEE Trans. Antennas Propagat., vol.60, no.12, pp.6023-6026, Dec.2012.

[4] S.B. Yeap, Z.N. Chen, X. Qing, "Gain-enhanced 60-GHz LTCC antenna array with open air cavities," IEEE Trans. Antennas Propag.,vol.59, no.9, pp.3470-3473, Sep. 2011.

[5] Y. She, R. Fujino, J. Hirokawa, M. Ando, D. Hanatani, M. Fujimoto, "LTCC oversized rectangular waveguide slot array antenna with airlayer in the radiating part in the millimeter-wave band," IEEE

[6] J. Xu, Z.N.Chen, X.Qing, W.Hong, "Bandwidth enhancement for a 60 GHz substrate integrated waveguide fed cavity array antenna on LTCC," IEEE Trans. Antennas Propag., vol.59, no.3, pp.826-832, March. 2011.

[7] M.J.Al-Hasan,T.A.Denidni, A.R.Sebak, "Millimeter-wave EBG-based aperture-coupled dielectric resonator antenna," IEEE Trans. Antennas Propagat., vol.61, no.8, pp.4354-4357, August 2013.

[8] M. Sun, Z.N. Chen,X.Qing, "Gain enhancement of $60-\mathrm{GHz}$ antipodal tapered slot antenna using zero-index metamaterial," IEEE Trans. Antennas Propagation., vol.61, no.4, pp.1741-1746, April 2013.

[9] G.H. Zhai,W. Hong, K. Wu, Z.Q. Kuai, "Wideband substrate integrated printed log-periodic dipole array antenna," IET Microwave Antennas Propagat., vol.61, no.6, pp.3009-3016, Feb 2010.

[10] R. Marqués, F. Mesa, J. Martel, and F. Medina, "Comparative analysis of edge- and broadside-coupled split ring resonators for metamaterial design-theory and experiments," IEEE Trans. Antennas Propag., vol. 51, no. 10 , pp. $2572-2581$, Oct. 2003

[11] R. Liu, A. Degrion, J.J. Mock, and D.R. Smith, "Negative index material composed of electric and magnetic resonators," Appl.Phys.Lett.,vol. 90, no. 26, Jun. 2007.

[12] X. Chen, T.M. Grzegorczyk, B.I. Wu, J. Pacheco Jr., J.A. Kong, "Robust method to retrieve the constitutive effective parameters of metamaterials," Phys. Rev. Lett., E 70, 016608, 2004.

[13] R.A. Alhalabi, G.M. Rebeiz, "High-gain Yagi-Uda antennas for millimeter-wave switched-beam systems," IEEE Trans. Antennas Propagat., vol.57, no.11, pp.3672-3676, Nov. 2009. 